\title{
Interfaces
}

INTERFACES Image Texte Language

$46 \mid 2021$

Jeux de Formats (2)

\section{Les otome games entre formatage idéologique et formation identitaire : une recherche-création en entreprise}

Hélène Sellier

\section{OpenEdition}

Journals

Édition électronique

URL : https://journals.openedition.org/interfaces/3849

DOI : $10.4000 /$ interfaces.3849

ISSN : 2647-6754

Éditeur :

Université de Bourgogne, Université de Paris, College of the Holy Cross

Référence électronique

Hélène Sellier, « Les otome games entre formatage idéologique et formation identitaire : une recherchecréation en entreprise », Interfaces [En ligne], 46 | 2021, mis en ligne le 15 décembre 2021, consulté le 16 juin 2022. URL : http://journals.openedition.org/interfaces/3849; DOI : https://doi.org/10.4000/ interfaces.3849

Ce document a été généré automatiquement le 16 juin 2022.

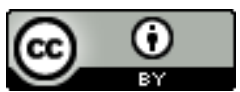

Les contenus de la revue Interfaces sont mis à disposition selon les termes de la Licence Creative Commons Attribution 4.0 International. 


\title{
Les otome games entre formatage idéologique et formation identitaire : une recherche-création en entreprise
}

\author{
Hélène Sellier
}

\section{Introduction}

1 Les otome games sont un genre médiatique qui se caractérise par la représentation d'une histoire d'amour entre une jeune fille, incarnée par la joueuse ${ }^{1}$, et un des nombreux hommes avec qui elle évolue. Le genre est né au Japon dans les années 1990 avant de se propager en dehors de l'Asie à la fin des années 2000. Sa popularité croissante ne peut se comprendre qu'en envisageant les contextes économique et culturel dans lesquels il est produit et consommé. En lien avec les incitations du gouvernement japonais à développer les nouvelles technologies dans les années 1990, le jeu vidéo a pris une part importante au sein des industries culturelles de l'archipel (Picard). Parmi les productions vidéoludiques, ont notamment été produits de nombreux jeux vidéo qui proposent un contenu érotique. Après la mise en place, dans les années 1990, de mesures gouvernementales de censure, les productions évoluent en jeux de drague (dating sim) et apparaît le genre du bishojo game, destiné à un public masculin et progressivement privé de tout contenu explicitement sexuel (Andlauer 2019a). Avec Tokimeki Memorial (Konami Digital Entertainment, 1994) ou plus récemment, Clannad (Key Entertainment, 2004), le public masculin est invité à retourner au temps de l'adolescence en gérant le quotidien d'une vie de lycéen et à séduire une des filles fréquentées. Le genre du bishojo game s'inscrit plus largement au sein de la culture otaku, qui désigne une réception fanesque des productions de l'industrie culturelle japonaise, en particulier les manga et les anime (Azuma) et qui se caractérise notamment par un attachement réel aux personnages fictionnels que Galbraith (2014) conceptualise en empruntant le terme moé aux communautés de fans. Le genre de 
l'otome game se développe dans cet environnement culturel, en réaction au bishojo game. En 1994, l'équipe de développement Ruby Party du studio Koei, entièrement féminine, produit sur Famicom un jeu pensé pour les filles: Angélique propose aux joueuses d'incarner une jeune fille qui a été choisie pour devenir reine du cosmos et qui est aidée par de jeunes hommes dont l'un peut devenir son amant. Dès leurs origines, les otome games reprennent certains marqueurs des bishojo games, en particulier la représentation de la séduction (Hyeshin). Ils s'inscrivent aussi dans la culture des productions japonaises destinées à des jeunes filles, identifiée sous le terme de shojo (Shamoon). Les otome games, comme les bishojo games, sont aussi parfois inclus dans le genre des Visual Novels. Cette catégorisation anglo-saxonne recouvre des œuvres diverses caractérisées par l'utilisation conjointe d'images en 2D représentant le décor et les personnages et de textes pour raconter une histoire (qui comporte parfois des choix). Comprenant dans certains cas des passages ludiques comme des énigmes, il peut s'agir de jeux de drague, mais d'autres thèmes sont aussi traités comme les intrigues policières (The Portopia Serial Murder Case, Enix, 1983), les mondes dystopiques (Snatcher, Konami, 1988) ou encore les jeux mortels (Zero Escape: Virtue's Last Reward, Chunsoft, 2012). Populaires au Japon sous différentes formes depuis les années 1990, ce type de productions japonaises, en particulier les otome games, n'arrivent sur le marché nord-américain et européen qu'à partir de la seconde moitié des années 2000. Leur lien avec la culture otaku explique la difficulté première d'un développement en dehors de l'Asie (Taylor 192) mais aussi leur succès récent. Les années 2010 marquent le début d'une abondance d'otome games en Occident, dans la continuité de la circulation médiatique des productions populaires japonaises amorcée dans les années 1990. Se développent notamment des pratiques de localisation et la conception de produits pour un marché international qui conduisent à une standardisation des productions (Andlauer 2019a). L'étiquette générique de l'otome game est aussi l'objet d'appropriations par des studios européens et américains qui revendiquent un héritage des productions japonaises (2019a) tout en introduisant des modifications dans les représentations de l'héroïne et des relations amoureuses (Morris). Ces changements s'expliquent en partie par les discours médiatiques qui accompagnent la réception des jeux japonais aux Etats-Unis et en Europe. La presse généraliste comme les magazines spécialisés sont en effet très critiques et questionnent notamment le caractère pornographique des productions et les stéréotypes du féminin et du masculin véhiculés (Bouvard et Triclot). Déployant le genre des jeux de drague, resté discret sur le marché occidental et intégré à l'ensemble plus large des «jeux pour fille» jusqu'à la fin des années 2000 (Andlauer 2019a), les récentes productions occidentales d'otome games s'appuient aussi sur des constructions socio-culturelles de la «romance» véhiculées notamment par les littératures populaires (comme les romans « à l'eau de rose ») et les cultures médiatiques (comme les comédies romantiques).

2 Puisque les otome games sont une désignation floue dont les manifestations sont multiples selon les contextes culturels, il est possible de comprendre cette catégorie comme un genre médiatique. Matthieu Letourneux considère ainsi que le genre n'est donc pas une entité unique, mais «le résultat d'un réseau complexe de discours en interaction et en tension dont les échanges et les contradictions produisent un effet d'unité problématique » (2016). Partager cette conception non-essentialiste des genres qui invite à les considérer dans leurs contradictions et leurs bricolages en fonction de leur contexte de création, production et réception ne signifie pas mettre de côté la question de leur forme. Plutôt que d'envisager un ensemble de caractéristiques fixes, il 
s'agit alors de repérer entre les différentes productions un «air de famille ». Cette notion de ressemblance de famille que Letourneux reprend à Wittgenstein $(2017,195)$ suppose que le genre autorise les variations entre les productions, donc que les otome games, tout en étant standardisés, autorisent foncièrement la transformation de leurs codes.

Dans une perspective de création artistique, les otome games peuvent aussi être envisagés comme un format au sens de "convention ou agencement de paramètres matériels, techniques et symboliques permettant de manière relativement stabilisée l'identification et l'évaluation des œuvres et autres artefacts dans un contexte culturel donné » (Zerbib 352). Cette approche permet d'aborder leur structure ludo-narrative mise en place au sein d'un programme informatique. La partie principale du jeu consiste en un ensemble de dialogues qui comprennent des choix. Que la joueuse soit obligée de sélectionner dès la fin de l'introduction le personnage masculin avec qui elle veut partager l'aventure ou que cette élection se fasse au fur et à mesure, chacune de ses réponses influe sur la relation avec le personnage qui est représentée quantitativement par une jauge, visible par la joueuse ou non. L'histoire diffère selon les décisions de la joueuse : non seulement, selon le niveau de la jauge, la joueuse aura accès à un récit plus ou moins romantique mais aussi, parfois, certains choix créent des embranchements narratifs catégoriquement différents (pour respecter une certaine cohérence). En parallèle du récit central, certains otome games, en particulier s'ils sont commercialisés sous la forme de free-to-play (c'est-à-dire avec un contenu majoritairement gratuit et des options payantes), contiennent d'autres éléments ludiques, comme la possibilité de customiser l'avatar, un système de classe (qui catégorise le personnage dans un groupe, comme les mages ou les guerriers, en fonction de ses caractéristiques déterminées par les activités effectuées), des jeux de hasard permettant d'avoir accès à des représentations graphiques supplémentaires ou encore un lieu de socialisation avec les autres joueuses. L'ensemble du dispositif construit la relation aux personnages et permet aux joueuses d'exprimer leur attachement à leur(s) être(s) virtuel préféré(s). L'expérience ludique, et en particulier les décisions stratégiques dans les moments de dialogues, semble construire l'idéal d'une bonne relation amoureuse où le personnage féminin a à cœur de séduire le personnage masculin qu'elle a élu. Cette représentation se matérialise notamment à la fin des scénarios qui, se divisant en plusieurs branches narratives, montrent la réussite ou l'échec du couple.

4 Genre médiatique et format artistique, les otome games permettent de s'interroger sur les normes sociales et esthétiques des productions culturelles, qui sont à la fois la condition d'un dialogue entre créateurs et récepteurs et le cadre qui influence les modalités et les contenus de ces échanges. Du point de vue de la réception, la forme des otome games, définie par des conventions culturelles autant que par des paramètres techniques, enferme-t-elle nécessairement les joueuses dans des fonctionnements sociaux établis, des modèles de relations hétérosexuelles où les femmes cherchent à plaire aux hommes? Du point de vue de la création, comment exploiter les logiques subversives qu'autorise le genre afin de créer une œuvre qui puisse, par sa forme, être identifiée comme otome game selon les conventions existantes sans inclure d'injonction normative?

5 Dans cet article, ces questions seront étudiées grâce à un travail de recherche-création (Gosselin; Paquin et Noury), et plus particulièrement sous deux modes identifiés par 
Chapman et Sawchuck (15-16) la recherche pour la création et la recherche à partir de la création. D'abord, la recherche est utilisée pour la création. Bien qu'il ne s'agisse pas d'une étape absolument antérieure à la création, elle amorce la phase de formation de l'objet. Les lectures théoriques sur les otome games sont utilisées pour se détacher des préconceptions sur les jeux de romance et comprendre la signification de ces productions en contexte. Ensuite, la création est le socle de la recherche. Cette dernière permet de tester des hypothèses. La conception de la structure narrative et l'écriture de dialogues d'un jeu de type otome game permet de présenter plusieurs pistes de réflexion sur la manière de construire un jeu qui laisse la possibilité de faire des expériences différentes et tente de ne pas contraindre la joueuse à adopter une morale ou un comportement.

6 Ce travail de recherche-création a été réalisé lors de la conception de la seconde partie du jeu Moonlight Lovers développé par le studio Beemoov et dont la parution a débuté en 2019. L'entreprise, fondée en 2006 par Jean-Philippe Tessier et Benoit Guihard à Nantes, connaît son succès initial avec le jeu de mode Ma Bimbo (2006) dont l'univers féminin est inspiré par le travail de Céline Ananian (Andlauer 2019a, 131). Depuis la sortie d'Amour sucré (2011), l'entreprise développe des stratégies éditoriales pour inscrire leurs créations sur le marché des jeux à destination d'un public féminin (258). Moonlight Lovers s'inscrit dans cette logique de production. L'avatar de la joueuse est une jeune fille orpheline qui hérite du manoir de ses parents pour ses dix-huit ans. Lorsqu'elle s'y rend pour la première fois, elle rencontre un groupe de six vampires qui en ont fait leur résidence. Un accident se produit, elle est gravement blessée et elle est obligée de choisir l'un d'entre eux avec qui elle partage un lien immuable de dépendance: lorsqu'un vampire fait d'un humain son calice, il ne peut plus se nourrir que du sang de ce dernier qui acquiert des pouvoirs mais perd une partie de sa liberté. À partir de l'introduction, le récit se sépare en six branches parallèles qui détaillent chacune l'histoire d'amour entre l'avatar de la joueuse et le vampire choisi.

7 Poursuivant les réalisations d'une précédente écrivaine, j'avais pour mission de créer le scénario de trois branches, appelées « routes » (celles de Aaron, Raphaël et Ethan), à partir de documents de design (qui contenaient parfois des propositions assez détaillées et d'autres des ébauches d'idées qui restaient à construire) et à écrire le texte du jeu (dialogue et narration). Mon travail consistait aussi à participer au choix de moments narratifs qui sont illustrés et à établir la progression de la jauge d'affection du vampire en fonction des choix de la joueuse. Puisque j'étais scénariste indépendante, ma production était soumise à l'approbation de la directrice de création (ChinoMiko, de son alias), responsable de la vision de l'ensemble du jeu. De plus, dans la mesure où ma collaboration a débuté alors que le projet était déjà en partie réalisé, je devais non seulement me conformer aux codes d'écriture du texte établis au sein du processus de production du studio, mais aussi respecter l'histoire déjà écrite, reprendre le fonctionnement de l'univers existant et réutiliser les ressources graphiques créées. Au format de l'otome game, se sont alors ajoutés d'autres cadrages qui ont influencé la création. Cependant, avec ces contraintes, notamment économiques, le projet de recherche-création a permis de faire émerger les propositions présentées dans cet article. 


\section{Conventions du genre de l'otome game}

8 Une description superficielle de la structure ludo-narrative de ces jeux, telle qu'elle a été faite précédemment, laisse penser que la joueuse serait, à son insu, incitée à se conformer à un modèle social. Par exemple, Love Letter From Thief $X$ (Voltage Inc, 2011) raconte l'histoire d'une jeune fille qui s'associe avec un groupe de voleurs pour trouver le chef-d'œuvre réalisé par son grand-père. Pendant l'introduction, l'héroïne choisit lequel des neuf personnages masculins veillera sur elle et l'histoire d'amour se construit à partir de cette décision. Le récit est séquencé pour mettre en valeur les étapes du couple: la rencontre, l'officialisation de la relation, les confidences, la proposition de mariage, la cérémonie... De plus, la joueuse doit sélectionner certaines réponses pour avoir accès à la meilleure fin possible.

Dans son article sur les dating-sim, Emily Taylor (205) soutient que les bishojo games dépeignent un désir des hommes de contrôler les femmes et constituent un moyen de réaffirmer la masculinité otaku. Ce regard masculin («male gaze ») se retrouverait aussi dans les jeux de dragues pour les filles. Plus largement, Sarah Christina Ganzon (244) écrit que les otome games reflètent des idéaux de genre essentialistes et ont tendance à conforter les joueuses au sein de modèles sociaux établis. Pour développer cette thèse, elle s'appuie sur une analyse des contenus des jeux du studio Cheritz et montre que les femmes sont confinées à l'espace domestique, encouragées à développer des activités traditionnellement féminines liées à l'apparence physique et incitées à faire des cadeaux à leur prétendant.

10 Ganzon note cependant qu'il existe des formes de réappropriation des jeux par les joueuses : «alors que ces jeux peuvent finalement devenir des outils idéologiques pour apprendre aux femmes à être des consommatrices dans les sociétés capitalistes, les pratiques internationales des fans d'otome games, de plus en plus importantes, ont le potentiel de résister à ces formes d'interpellation. $»^{2}$ (245) Dans la lignée des Cultural Studies qui s'intéressent notamment aux réceptions des objets culturels institutionnellement non-légitimés, Ganzon souligne l'importance de comprendre les otome games en fonction des utilisations des joueuses, qui sont de l'ordre du détournement ou du "braconnage", défini par Michel de Certeau comme une appropriation créative d'un contenu (239-255). Ces productions autorisent la constitution d'un espace social où peuvent s'exprimer les désirs féminins. Dans sa communication «Becoming an avatar in a Japanese Love Game: Female Identity and Desired Alienation", Agnès Giard s'intéresse plus particulièrement aux joueuses d'otome games qui considèrent un personnage fictionnel comme leur amant, appelées yume joshi. La chercheuse montre que cette relation leur permet de se libérer de la crise du système matrimonial au Japon tout en construisant collectivement l'identité de femmes qui vivent pour elles-mêmes en prenant en compte leurs désirs. Giard interprète cette réception des jeux comme une réappropriation. Elle affirme en effet que la structure ludo-narrative renforce les pires stéréotypes de genre : «L'histoire et les mécaniques de jeu incitent les joueuses à penser que la seule manière d'avoir de la valeur consiste à faire plaisir à un personnage masculin et que la seule manière de lui faire plaisir consiste à se faire belle $»^{3}$. Cependant, contrairement à Ganzon et Giard, il est possible de soutenir que l'empowerment permis par otome games (au sens d'un processus socio-culturel qui se matérialise à la fois de manière individuelle comme 
prise de confiance en soi et de manière collective par un pouvoir d'agir (Bacqué et Biewener) ne se limite pas aux pratiques d'appropriation et de résistance des fans.

11 Certaines recherches sur les jeux de drague proposent une interprétation différente des éléments narratifs présents dans les œuvres. En ce qui concerne les bishojo games, Azuma (59) et Galbraith (2011) montrent tous les deux que ce genre de jeux ne doit pas être interprété comme une réaffirmation de la masculinité. Galbraith souligne en effet que le joueur s'identifie davantage avec les personnages non-joueurs féminins qu'avec son avatar masculin puisque leurs corps sont particulièrement expressifs. La logique des bishojo games ne repose pas sur un désir de possession et de satisfaction sexuelle, mais sur une projection de soi sur des corps émotionnées ("soulful bodies»). Azuma (59) avait déjà souligné la féminité des bishojo games en expliquant que le but de ces productions était de permettre aux joueurs de ressentir des émotions et de se laisser aller à pleurer, en supposant que ce comportement est, dans nos sociétés contemporaines, attribué à des attitudes féminines. Les bishojo games se démarquent en ce sens de nombreuses productions vidéoludiques populaires qui mettent en scène une «masculinité militarisée » (Genvo 2008). Julien Bouvard et Matthieu Triclot étudient la tension au sein du genre entre la "pornographie empathique " permise par le «male gaze » qui encourage le voyeurisme des corps féminins et « une opération de projection affective qui consiste à se mettre à la place du personnage émotionné » (162). Ces recherches soulignent ainsi la complexité des mouvements de résistance et de reproduction des stéréotypes de genre au sein des œuvres.

Envisager les bishojo games comme des productions qui ne renforcent pas nécessairement les normes sociales de genre existantes invite à interpréter les otome games autrement que comme un cadre normatif du féminin. Cette lecture des jeux de drague destinés à un public féminin semble d'autant plus pertinente que le genre se construit en réaction aux bishojo games et en tension avec les clichés qu'ils intègrent et détournent. S'intéressant à la construction historique de la culture shojo, Deborah Shamoon décrit la manière dont l'imaginaire féminin se construit à partir d'une représentation de l'amitié féminine, à la fois jugée comme innocente et permettant l'expression des désirs des jeunes filles (10-13). Les otome games constituent de même un endroit sécurisé pour que les femmes fassent l'expérience de leurs fantasmes et de leurs identités et permettent l'extension de la culture féminine au sein des jeux vidéo (Hyeshin 188). Dans une étude portant sur les otome games représentant des périodes historiques, Kazumi Hasegawa (136) montre que si les jeux semblent dans un premier temps nationalistes et hétéronormés, le récit d'un amour avec une figure historique permet aux joueuses « d'imaginer leur place dans le passé et de produire des histoires alternatives $»^{4}$ Il s'agit alors de défier les constructions identitaires contraintes, notamment les normes de genre, par un acte de «queering history ». En ce sens, les otome games fournissent un contexte approprié pour faire une expérience active ("agency») et en toute sécurité de son identité et de sa sexualité. Leticia Andlauer (2019b) souligne aussi la manière dont ces productions construisent progressivement une nouvelle esthétique du romantisme: le choix de l'amant par la joueuse, la possibilité qu'offrent certains jeux de poursuivre différentes relations ou encore la représentation d'un personnage masculin attentif aux envies de l'avatar féminin sont des moyens de construire des rôles de genres différents des représentations traditionnelles de l'amour hétérosexuel. Au cœur des otome games, comme du roman « à l'eau de rose ", se trouvent le plaisir et le désir féminin. 
13 sociaux établis, les otome games sont reçus et interprétés comme une expérience formative où les joueuses apprennent à la fois à maîtriser les imaginaires sociaux et à trouver leur position par rapport aux conventions sociales. Si la forme actuelle ouvre ces possibilités, les critiques soulignent souvent le caractère progressif de l'inclusion d'éléments ludo-narratifs au sein des otome games remettant en question les rôles de genre dans les relations hétérosexuelles. Par exemple, même si la joueuse peut choisir le personnage masculin avec lequel l'héroïne vivra une histoire d'amour, cette dernière est souvent dépeinte comme moins expérimentée que l'homme qu'elle désigne comme mentor. Dans Love Letter From Thief X (Voltage Inc, 2011), elle doit se familiariser avec le monde des voleurs et en apprendre plus sur son grand-père. Andlauer $(2019 b, 361)$ écrit ainsi qu'il existe une "dissonance entre les histoires d'amour romantiques et hétéronormatives et les dynamiques de genre inscrites dans la structure du jeu et au sein des pratiques fanesques $»^{5}$. Dans une perspective de création, la question se pose alors des formes d'une production qui, tout en s'inscrivant dans la forme standardisée de l'otome game, pourrait moduler le genre, exploiter la plasticité générique, afin d'en faire émerger d'autres possibilités.

\section{Modulations créatives du format de l'otome game}

14 Comprendre la réception des otome games en lien avec différents contextes socioculturels invite à se départir des préconceptions sur le format, c'est-à-dire, au sein du processus créatif, à remettre en jeu des conventions, à déstabiliser les agencements, en exploitant la malléabilité du genre et en réfléchissant à un récit qui tente de ne pas imposer un cadre moral particulier à la joueuse. Par rapport aux représentations des relations amoureuses, il s'agit alors de ne pas contraindre les joueuses dans des comportements traditionnellement associés au féminin, ni de les forcer à adopter des attitudes de résistance par rapport à la norme. La notion de jeux expressifs, créée par Sébastien Genvo à partir de deux processus de recherche-création, Keys of a gamespace (2011) et Lie in my heart (2019), est à ce titre particulièrement utile. Le chercheur propose la définition suivante : «[...] un jeu expressif peut se définir, du point de vue de la conception, comme un jeu qui incite à se mettre à la place d'autrui pour explorer ses problèmes psychologiques, sociaux, culturels. En tant que jeu, il va confronter le joueur aux choix de vie et dilemmes que ces problèmes créent. Il s'agit donc à la fois d'exprimer une problématique individuelle ou sociale tout en ouvrant en retour la possibilité au joueur de s'exprimer sur celle-ci.» $(2019,133)$ Les jeux expressifs ne cherchent pas à convaincre, à persuader, à expliquer ou à transmettre une compétence mais ont pour objectif d'ouvrir un questionnement et de permettre à la joueuse de s'exprimer sur une thématique en incluant dans le système ludo-narratif différentes «morales non-moralisantes» $(2019,133)$. Le jeu expressif propose ainsi des moments de choix et la représentation de leurs conséquences sans apporter de conclusion sur le fait qu'ils soient bons ou non. Bien que Moonlight Lovers ne soit pas un jeu expressif, puisqu'il ne partage pas toutes les caractéristiques mises en lumière par la définition, le travail que j'ai réalisé sur le jeu a pour ambition de laisser une place à l'expression de la joueuse. Plus précisément, trois pistes ont été travaillées: la relation à l'avatar, la représentation de la relation amoureuse et des amitiés et la structure narrative.

Interfaces, 46 | 2021 
Contrairement à la majorité des otome games qui s'appuient sur des avatars construits sur le modèle de la « coquille vide » (Taylor 198), c'est-à-dire qui présentent le moins de caractéristiques définis possibles et aucune personnalité, et dans la lignée des modifications du format réalisées dans les productions occidentales (Morris), j'ai fait en sorte que le personnage-joueur prenne vie grâce à l'interprétation de la joueuse. L'histoire créée ne laissait pas beaucoup de place au passé de l'héroïne puisqu'elle est orpheline et arrive dans un nouveau monde dont elle ne connait rien. Cependant, pour favoriser l'identification et étoffer le personnage, je me suis appuyée sur des souvenirs d'enfance qui sont de l'ordre d'expériences communes, comme des souvenirs de maladie, de disputes avec des amis, de moments d'apprentissage de leçons. Dans le premier chapitre de la route d'Ethan, l'héroïne ne se souvient pas d'être arrivée au manoir lorsqu'elle se réveille, la joueuse a alors le choix de se lever immédiatement ou non. L'option de la paresse renvoie à une situation courante au lycée :

CHOIX

Héroïne: (Pas le courage de regarder l'heure, je suis sûre que je suis à la bourre de toute manière.)

Héroïne: (Ça doit être M. Zibrowski, en plus, ce matin... Vraiment pas le courage...)

[...]

Narrateur: De temps en temps, je sentais à travers mes paupières closes les rayons du soleil qui chatouillaient mon visage et je me replongeais avec délice dans l'obscurité.

[...]

Narrateur: Je me réveillai en sursaut et cherchai déjà ma liste d'excuses pour justifier ma grasse matinée.

Evoquer des situations habituelles ou raconter des sensations plutôt que décrire précisément une anecdote permet de laisser à la joueuse la possibilité de compléter les scènes avec ses propres références. Contrastant fortement avec les dialogues par l'utilisation d'une temporalité passée et réflexive ainsi que l'usage important de figures de style, la voix narrative qui représente la vie intérieure de l'héroïne invite la joueuse à explorer ses propres émotions en les comparant à celles que le texte décrit. Les réflexions de l'héroïne sont souvent écrites sous la forme de questions, comme lorsqu'elle s'interroge sur les premières émotions qu'elle ressent après sa rencontre avec Raphaël :

Narrateur: Mon cerveau tentait d'analyser la source de ma joie infatigable. N'étaitce pas ce que j'avais toujours voulu ? Toujours espéré ? Une nouvelle vie. Quelqu'un avec qui la partager.

17 Les hésitations de l'héroïne sont aussi utilisées pour donner la place à l'interprétation de la joueuse. Par exemple, le constat d'un climat de tension entre les vampires, au début de la route d'Ethan, est l'occasion de laisser la joueuse réfléchir à la stratégie qu'elle veut adopter face à ce personnage particulièrement odieux :

Héroïne: (Contrairement à ce qu'on me répète depuis que je suis arrivée, tout le monde n'est pas copain, là-dedans. Je l'ai bien vu...)

CHOIX

Héroïne: (Il va falloir que je trouve ma place, que je m'impose...)

CHOIX

Héroïne: (Peut-être que si je gagne de l'influence, je trouverai un moyen de partir...)

CHOIX

Héroïne: (Jouer le jeu, gagner leur confiance jusqu'à ce que je puisse me débarrasser d'Ethan.) 

défi par rapport aux actions racontées. L'héroïne devait pouvoir être comprise comme un personnage fort, qui ne soit pas soumise ou docile, sans que le jeu fasse pression sur la joueuse pour que les réponses données soient toujours héroïques ou rebelles. Il était important que les comportements de préservation, d'évitement ou encore d'hésitation soient présentés comme valides. Pour cela, j’ai décidé que les choix donnés à la joueuse ne seraient pas évidents. Par exemple, dans le troisième chapitre de la route d'Ethan, l'héroïne se retrouve face à Ivan, le vampire qui est responsable de sa chute et de sa condition de calice. Le voyant blessé, l'héroïne doit choisir d'aider ou non son agresseur qu'elle sait être un vampire. Invitée à avoir une réflexion morale, la joueuse doit donc décider si elle souhaite pardonner un être qui l'a blessée et secourir une créature qu'elle juge sanguinaire. Faire preuve d'indulgence n'est pas présenté unilatéralement comme la bonne solution dans la mesure où la réaction d'Ethan est négative dans ce cas.

De la même manière que la personnalité de l'avatar cherche à s'adapter en fonction de l'interprétation de la joueuse, la relation avec le personnage masculin choisi ne se veut pas unidirectionnelle. Par rapport aux remarques de Giard (2020), être d'accord avec lui, chercher à lui faire plaisir à tout prix ou ne se concentrer que sur l'apparence de son avatar n'est pas nécessairement la meilleure option pour que se construise le couple. La liaison entre les deux personnages grandit lorsque les actions de l'héroïne sont conformes aux valeurs morales du vampire. Par exemple, Aaron, l'un des deux vampires que la joueuse peut choisir en début de partie, estime l'action courageuse tandis que Raphaël, lui, préfèrera la prudence réfléchie. Dans chacune de leur route, est représentée une leçon d'escrime qui sert d'entraînement dans un monde dangereux: alors qu'Aaron apprécie davantage l'hérö̈ne si la joueuse prend la décision d'agir de façon un peu risquée, Raphaël la jugera imprudente si un choix similaire est fait. Un système de points est utilisé, mais un travail a été mené pour que tous les chemins narratifs soient intéressants. Par exemple, dans le chapitre 7 de la route de Raphaël, l'héroïne a le choix entre lire un livre conseillé par Raphaël (ce qui renforce immédiatement leur relation) ou s'entraîner avec Aaron (ce qui a pour conséquence immédiate d'irriter Raphaël, mais qui est utile pour l'aider au moment du combat final). Même si la relation n'est pas de l'ordre de l'amour passionnel, elle est dépeinte comme un échange humain dont les nuances et la complexité forment la richesse. Dans la route de Ethan, j'ai ainsi donné le choix à la joueuse de ne pas jouer une relation amoureuse.

Au sein des représentations des relations hétérosexuelles dans la culture médiatique (Williams), et notamment dans les otome games, les scènes de sexe constituent un moment important du récit, en tant que matérialisation du lien entre les personnages. Cependant, de nombreuses histoires d'amour vidéoludiques, issues de contextes socioculturels différents et à destination de publics variés, sont construites sans référence à la sexualité (Bouvard et Triclot). Pour ne pas imposer d'épisode érotique à la joueuse qui ne le souhaiterait pas, je me suis assurée de clarifier l'orientation que prend le récit dans ces cas. Par exemple, dans le chapitre 8 de la route d'Aaron, alors qu'il y a une tension sexuelle entre les personnages, le vampire demande à l'héroïne si elle est sûre de vouloir aller plus loin. Si la joueuse refuse, elle a accès un moment intime où les amoureux lisent ensemble. Dans la lignée des otome games qui mettent en avant le désir féminin, j'ai aussi choisi de refuser toute représentation idéaliste tout en privilégiant la description de sensations et d'émotions. Ceci signifie notamment écrire les hésitations, 
les maladresses, les frustrations, les ratés et mettre l'accent sur le plaisir corporel plutôt que sur les sentiments amoureux. Dans les fictions médiatiques populaires, les femmes sont souvent représentées comme passives. Plutôt que de forcer la joueuse à s'identifier à une attitude ou une autre, j'ai inclus des moments de choix au sein des moments intimes, comme dans cet extrait du le chapitre 6 de la route de Raphaël :

Narrateur: Un instant après, il passa sa main sous mon menton et ses doigts commencèrent à courir doucement le long de ma gorge, le long de mes épaules.. Narrateur: Pour enfin plonger ensuite dans l'interstice entre le tissu et ma peau.

CHOIX

Héroïne: (Cette vague qui monte en moi... La laisser m'envahir...)

CHOIX

Héroïne: (Cette envie de passer ma main sur lui moi aussi...)

CHOIX

Héroïne: (Cette envie de sentir toute sa peau contre la mienne, d'arracher ses vêtements...)

21 J'ai ainsi cherché à concevoir l'expressivité du jeu (au sens de Genvo) afin qu'elle ne se limite pas aux interprétations des situations ou aux stratégies à adopter pour résoudre des problèmes mais qu'elle se matérialise aussi dans les moments clefs des fictions sentimentales que sont les relations intimes.

En parallèle des scènes de sexe, les passages où sont décrites les morsures des vampires participent à la construction de la relation entre l'héroïne et le personnage masculin et symbolisent souvent dans la culture médiatique la sexualité (Baussand). Les représentations varient d'un vampire à un autre, mais j'ai été attentive à les utiliser sans jamais mettre la joueuse en position de devoir s'identifier avec un personnage qui est agressé. Dans la majorité des cas, les morsures sont accordées volontairement et permettent à l'héroïne d'asseoir son pouvoir sur le vampire. Par exemple, dans le chapitre 3 de la route d'Ethan, la joueuse a le choix de proposer une morsure au vampire ou non. Il s'agit de représenter les tentatives de l'hérö̈ne de se placer en position de force en régulant l'accès à la force vitale qu'elle représente pour Ethan. Quand la morsure n'est pas une décision de l'héroïne, la scène est explicitement écrite comme un moment difficile, et lorsqu'il s'agit de la représentation symbolique d'un viol (comme la transformation en calice dans la route d'Ethan), l'extrait mentionnant le souvenir est entièrement optionnel et la joueuse peut décider de quitter la scène à plusieurs reprises. Bien que ces techniques narratives ne viennent pas palier la logique du récit sentimental de vampire dans lequel l'héroïne humaine est d'abord en position de faiblesse face à la créature, elles ont pour but de ne pas imposer à la joueuse un récit violent et de lui offrir un cadre narratif sécurisant dans lequel l'inégalité des positions ne suppose pas nécessairement de devoir subir un rapport de domination.

Même si un vampire est choisi dès le départ comme potentiel amant, j'ai essayé de ne pas utiliser les autres personnages uniquement comme moyen de mettre en lumière ce protagoniste masculin. La joueuse ne peut pas interagir autant avec tous les vampires au sein de chaque scénario, mais les personnages secondaires ne sont pas interchangeables et l'héroïne peut développer des amitiés. Certaines conversations portent alors sur d'autres sujets que sa relation amoureuse, notamment des scènes de la vie quotidienne. Par exemple, la joueuse se rend dans la bibliothèque au milieu du chapitre 4 de la route d'Aaron, elle pourra surprendre une conversation où un jeune vampire tente de convaincre un autre habitant du manoir plus âgé et réfractaire aux technologies modernes d'essayer les livres audio. D'autre part, bien que le jeu ne 
cherche pas à représenter directement des problématiques socio-culturelles, contrairement aux jeux expressifs (Genvo), ces questionnements sont présents par moments. Par exemple, dans le troisième chapitre de la route de Raphaël, une conversation autour de la grossesse permet à la joueuse d'exprimer ses désirs sans qu'elle soit jugée. De même, bien que le format de l'otome game incite à écrire uniquement un amour hétérosexuel exclusif, un des scénarios offre la possibilité à la joueuse d'expérimenter une autre forme de relation: une des fins de la route d'Aaron représente l'héroïne qui développe des sentiments amoureux pour le personnage masculin mais aussi une amie, Farah, et laisse ouverte la possibilité d'un trouple.

Les expérimentations autour de l'agentivité de la joueuse ne concernent pas seulement la définition de l'avatar ou la construction des relations aux personnages secondaires, mais aussi la structure ludo-narrative. Bien que chaque choix donné ne puisse pas créer une branche narrative indépendante et que le design narratif des productions interactives repose essentiellement sur des formes de goulot d'étranglement (Ashwell), j'ai décidé d'écrire de nombreux moments de décision, pour laisser la place à l'expression de la joueuse (entre vingt et trente par chapitre en moyenne). De plus, en encourageant l'exploration, qui est récompensée par des scènes optionnelles, j'ai essayé d'inciter la joueuse à développer sa confiance en ses propres compétences. Parfois, plutôt que de suivre les indications explicitement données par le texte, il est possible de trouver une autre solution par rapport à la situation présentée et cette voie donne un bonus au sein du système numérique. Par exemple, dans la route d'Aaron, lorsqu'un meurtre se produit, le vampire demande à l'héroïne de rejoindre sa chambre. Il est possible de comprendre cette indication comme une consigne du jeu. Cependant, si la joueuse se rend dans une autre pièce, explorant l'espace, elle est récompensée par un moment avec Aaron. Ce principe qui consiste à demander à la joueuse de faire des actions très spécifiques pour découvrir certains secrets du jeu est celui qui sous-tend les premiers Easter Eggs ces références cachées (sous forme d'une image, d'un message...) qui forment un code vidéoludique particulièrement ancré dans cette culture.

\section{Conclusion}

Bien que les otome games soient pris dans un réseau de contraintes techniques et de conventions culturelles, le format constitue un cadre, partagé par des communautés, qui permet aux joueuses de se construire. Du côté de la création, c'est parce que le format, tout en étant « relativement stabilisé » (Zerbib), n'est pas figé qu'il constitue un terreau fertile. Conçues à partir de la recherche sur le genre, les tentatives pour créer un objet qui laisse une marge de manœuvre à la joueuse pour faire l'expérience de son identité, pour exprimer ses désirs et tester la relation amoureuse dans un environnement sécurisé ont été fructueux dans le processus de la création dans la mesure où ils ont orienté, pour l'instant, l'écriture de trois routes, soit plus de trente mille mots. Ces scénarios ont, en retour, permis d'expérimenter des stratégies narratives qui laissent la place à l'expression de la joueuse. Il s'agit par exemple d'encourager à la fois l'identification et la réflexivité par rapport à l'avatar et de multiplier les choix dans des situations diverses, qu'ils créent ou non un embranchement réel dans la structure narrative. Il serait alors intéressant de mener une étude de la réception du jeu pour évaluer l'effet réel de ces tentatives d'écriture, 
pour déterminer si les textes ont été interprétés par les joueuses tels qu'ils ont été imaginés. L'absence de cette analyse constitue une des limites de ce travail de recherche-création, au même titre que l'impossibilité légale d'étudier le processus de création au sein d'une approche d'autopoïetique qui s'intéresse au processus de création de son propre objet (Lelièvre). Cependant, ces questionnements seront développés dans le cadre de mon travail pour The Seed Crew, un studio de jeu vidéo indépendant dont le premier jeu, RecovR, explore les problématiques liées à la diversité, l'inclusion et la mixité et au sein duquel je suis missionnée pour faire de la recherchecréation.

\section{BIBLIOGRAPHIE}

\section{Ouvrages cités}

ANDLAUER, Laeticia. La construction adolescente au regard des pratiques et productions de l'industrie de l'otome game en France. Thèse de doctorat, Université de Lille, 2019a.

ANDLAUER, Laeticia. " Pursuing One's Own Prince: Love's Fantasy in Otome Game Contents and Fan Practice ». Mechademia Second Arc: Childhood. vol. 11.1. University of Minnesota Press. 2019b : 166-183

ASHWELL, Sam. "Standard Patterns in Choice-Based Games », Blog These Heterogeous Tasks, 2015. https://heterogenoustasks.wordpress.com/2015/01/26/standard-patterns-in-choice-basedgames/

AZUMA, Hiroki. Génération otaku - les enfants de la postmodernité. 2008. Paris: Hachette. BAUSSAND, Maël. « Dracula : mécanique des fluides et roman des angoisses circulatoires ». GLAD!. n 5. 2018. https://www.revue-glad.org/1248

BACQUE, Marie-Hélène et Carole BIEWENER « L'empowerment, un nouveau vocabulaire pour parler de participation? ", Idées économiques et sociales, vol. 173, no. 3, 2013 : 25-32.

BOUVARD Julien et Mathieu TRICLOT. « Les 17 ans éternels : apprendre à jouer à Clannad ».Jeu vidéo et adolescence. 2019. Ed. Vincent Berry et Leticia Andlauer. Presses de l'Université de Laval. 149-176.

CHAPMAN, Owen et Kim SAWCHUCK. « Research-Creation: Intervention, Analysis and "Family Resemblances"». Canadian Journal of Communication, n 37. 2012: 5-26.

DE CERTEAU, Michel. L'invention du quotidien I. Arts de faire, 1990. Paris : Gallimard.

GALBRAITH, Patrick. « Bishōjo Games: ‘Techno-Intimacy’ and the Virtually Human in Japan ». Game Studies. vol. 11, n², 2011.

GALBRAITH, Patrick. Otaku Spaces. 2012. Seattle: Chin Music Press.

GALBRAITH, Patrick. The Moe Manifesto: An Insider's Look at the Worlds of Manga, Anime, and Gaming, 2014. North Clarendon: Tuttle Publishing. 
GANZON, Sarah Christina. « Sweet solutions for female gamers: Cheritz, Korean otome games and global otome game players ». Digital love: Romance and sexuality in games. 2018. Ed. in Heidi Macdonald. London, England: Taylor and Francis. 225-244.

GENVO, Sébastien. « Comprendre et développer le potentiel expressif ». Hermès, La Revue. vol. 62. $\mathrm{n}^{\circ} 1.2012: 127-133$.

GENVO, Sébastien. «Faire jouer la vie d'autrui : enjeux ludo-narratologiques du jeu autobiographique Lie in my heart », Communication lors du colloque Lusor in Fabula, 2019. https:// webtv.univ-rouen.fr/videos/04-a-lusor-in-fabula-faire-jouer-la-vie-dautrui-enjeux-ludonarratologiques-du-jeu-auto-biographique-lie-in-my-heart-par-sebastien-genvo/

GIARD, Agnès. « Becoming an avatar in a Japanese Love Game : Female Identity and Desired Alienation ». Communication lors de la conférence Internationale en ligne « Desired Identities. New Technology-based Metamorphosis in Japan ». 2020. https://www.youtube.com/watch? $\underline{\mathrm{v}=\mathrm{Q} 4 \_1 \mathrm{wk} 4 \mathrm{TuRE}}$

GOSSELIN, Pierre. «La recherche en pratique artistique ». La recherche création. Pour une compréhension de la recherche en pratique artistique. 2006. Ed. Gosselin Pierre et Le coquiec Eric. Québec : Presses de l'Université du Québec.

HASEGAWA Kazumi. « Falling in love with history: Japanese girls' otome sexuality and queering historical imagination ». Playing with the Past: Digital Games and the Simulation of History. 2013. Ed. M. W. Kapell and A. B. Elliot. London : Bloomsbury. 135-150.

HYESHIN, Kim. « Women's games in Japan: Gendered identity and narrative construction ». Theory, Culture \& Society, vol. 26, 2009. 165-188.

LELIEVRE, Edwige, « Research-creation methodology for game research ». 2018. https:// hal.archives-ouvertes.fr/hal-02615671/document

LETOURNEUX, Matthieu. « Le genre comme pratique historique ». Belphégor. n 14. 2016. https:// belphegor.revues.org/732\#entries

LETOURNEUX, Matthieu. Fictions à la chaîne. Littératures sérielles et culture médiatique. 2017. Paris : Seuil.

PICARD, Martin. "The foundation of geemu: A brief history of early Japanese video games ». Game Studies. $\mathrm{n}^{\circ}$ 13. 2013. http://gamestudies.org/1302/articles/picard

MORRIS, Lucy. « Love Transcends All (Geographical) Boundaries The Global Lure of Romance Historical Otome Games and the Shinsengumi ». Digital Love : Romance and Sexuality in Video Games. 2018. Ed. Heudi McDonald. Boca Raton : CRC Press.

PAQUIN Louis-Claude et Cynthia NOURY. « Cartographies de la recherche-création » ? 2018 http://lcpaquin.com/cartoRC/

SHAMOON, Deborah. Passionate Friendship: The Aesthetics of Girl's Culture in Japan. 2012. Honolulu: University of Hawaii Press.

TAYLOR, Emily. « Dating-Simulation Games: Leisure and Gaming of Japanese Youth Culture ». Southeast Review of Asian Studies. vol. 29. 2007. 192- 208.

WILLIAMS, Linda. Screening Sex. Une histoire de la sexualité sur les écrans américains depuis les années 1960. 2014. Nantes : Éditions Capricci.

ZERBIB, David. «L'Ère du format ». In Octavo, Des formats de l'art. 2015. Ed. David Zerbib. Dijon et Annecy : Les Presses du réel. 


\section{NOTES}

1. Dans cet article, le féminin neutre est employé pour désigner le public des otome game, qui est majoritairement féminin, et le masculin neutre pour faire référence au public des bishojo games, qui sont destinés à un public masculin. Ces usages ne présument pas du genre de chacune des personnes qui jouent à ces jeux.

2. [«while these games can ultimately become ideological tools to train women as consumers in capitalist societies, the otome games' growing international fandom has a potential to resist these forms of interpellation »]. Toutes les traductions au sein de cet article sont le fait de l'auteure.

3. [«The narrative and game mechanics induce players to think that the only way to get value is to please a male character and the only way to please him is to make themselves beautiful »] (Giard, 2020)

4. [« imagine their space in the past as well as to produce alternative narratives »]

5. ["reveals a dissonance between the heteronormative fairy tales of love and the gendered dynamics of the actual game structure and fan practices »]

\section{RÉSUMÉS}

Cet article interroge le genre médiatique et le format artistique des otome games, jeux vidéo qui racontent des histoires d'amour destinées à un public féminin. Un projet de recherche-création ancré dans le fonctionnement économique des industries culturelles permet de montrer que ces productions vidéoludiques standardisées permettent paradoxalement aux joueuses d'expérimenter leur identité et autorisent les variations en partant de l'idée centrale du désir féminin.

This article questions the media genre and artistic format of otome games, video games based on love stories and aimed at female audiences. A research-creation project rooted in the economic working of cultural industries has shown that these standardized video games paradoxically allow players to experiment with their identity and allow variations around the central concept of female desire.

\section{INDEX}

Keywords : research-creation, otome game, dating simulation, expressive game, popular culture, media studies

Mots-clés : recherche-création, otome game, jeu de romance, jeu expressif, culture médiatique 


\section{AUTEUR}

\section{HÉLÈNE SELLIER}

Membre de l'OMNSH et de l'association LPCM, chargée de recherches dans le studio The Seed

Crew

Hélène Sellier est docteure en Littérature Comparée. Elle a soutenu sa thèse intitulée

"Littérature et jeux vidéo : représentations réciproques » et dirigée par Irène Langlet en septembre 2019 à l'Université Paris-Est Marne-la-Vallée. Elle a notamment contribué au recueil Littératures du jeu vidéo dirigé par Paul-Antoine Colombani, Guillaume Grandjean et Siegfried Würtz (2019) ainsi qu'à l'ouvrage Jeu vidéo et livre dirigé par Fanny Barnabé et Bjorn-Olav Dozo (2015). En parallèle, elle est Narrative Designer dans l'industrie du jeu vidéo et enseigne les cultures médiatiques et l'écriture de récits numériques dans plusieurs universités, en France et au Royaume-Uni. 